\title{
Targeted delivery of propionate to the human colon prevents body weight and intra-abdominal adipose tissue gain in overweight adults
}

\author{
E. S. Chambers ${ }^{1}$, A. Viardot ${ }^{1}$, A. Psichas ${ }^{1}$, D. J. Morrison ${ }^{2}$, K. G. Murphy ${ }^{3}$, \\ S. E. K. Zac-Varghese ${ }^{3}$, K. MacDougall ${ }^{4}$, T. Preston ${ }^{2}$, M. C. Tedford ${ }^{4}$, J. D. Bell ${ }^{5}$, \\ E. L. Thomas ${ }^{5}$, S. Mt-Isa ${ }^{6}$, D. Ashby ${ }^{6}$, W. S. Dhillo ${ }^{3}$, S. R. Bloom ${ }^{3}$, W. G. Morley ${ }^{7}$, S. Clegg $^{7}$ \\ and G. Frost ${ }^{1}$ \\ ${ }^{1}$ Nutrition and Dietetic Research Group, ${ }^{2}$ Scottish Universities Environmental Research Centre, University of Glasgow, \\ G75 OQF, UK, ${ }^{3}$ Section of Investigative Medicine, Imperial College London, W12 ONN, UK, ${ }^{4}$ School of Science, \\ University of the West of Scotland, Paisley PA1 2BE, UK, ${ }^{5}$ MRC Clinical Science Centre, Imperial College London, \\ W12 ONN, UK, ${ }^{6}$ Imperial Clinical Trials Unit, Imperial College London, W2 $1 N Y$, UK and ${ }^{7}$ Leatherhead Food \\ Research, Leatherhead KT22 7RY, UK
}

The colonic microbiota ferment available carbohydrates, producing short chain fatty acids (SCFAs), which trigger the release of the anorectic gut hormones peptide YY (PYY) and glucagon like peptide-1 (GLP-1) by stimulating the free fatty acid receptors (FFAR) 2 and 3 on the enteroendocrine $\mathrm{L}$ cells ${ }^{(1)}$. We have developed a novel inulin propionate ester that targets delivery of gram quantities of the SCFA propionate to the human colon and which significantly increases PYY and GLP-1 secretion and reduces food intake following acute ingestion at a dose of $10 \mathrm{~g}$ (unpublished observation). We hypothesised that long-term supplementation with propionate ester would prevent weight gain in overweight adults.

Forty nine men and women aged 40-65 years, with a body mass index (BMI) of $25-40 \mathrm{~kg} / \mathrm{m}^{2}$ were recruited and randomly assigned to receive either $10 \mathrm{~g} /$ day inulin control $(n=24)$ or $10 \mathrm{~g} /$ day propionate ester $(n=25)$ for 24 -weeks. Body composition data, assessed using magnetic resonance imaging, was collected from 17 subjects in the propionate ester group and 15 subjects in the control group.

\begin{tabular}{|c|c|c|c|c|c|c|c|c|c|c|}
\hline \multirow[b]{3}{*}{ AT Distribution ( $\%$ Total AT) } & \multicolumn{4}{|c|}{ Control $(n=15)$} & \multicolumn{4}{|c|}{ Propionate Ester $(n=17)$} & & \\
\hline & \multicolumn{2}{|c|}{ Week 0} & \multicolumn{2}{|c|}{ Week 24} & \multicolumn{2}{|c|}{ Week 0} & \multicolumn{2}{|c|}{ Week 24} & \multicolumn{2}{|c|}{ Propionate Ester-Control } \\
\hline & Mean & $\overline{\mathrm{SE}}$ & Mean & $\mathrm{SE}$ & Mean & $\overline{\mathrm{SE}}$ & Mean & $\mathrm{SE}$ & Mean & $95 \% \mathrm{CI}$ \\
\hline Subcutaneous AT & 81.3 & 1.9 & 80.6 & $1.8 * *$ & 76.3 & 1.7 & 76.0 & 1.6 & -0.59 & $1.69,0.50$ \\
\hline Internal AT & 18.7 & 1.9 & 19.4 & $1.8^{* *}$ & 23.7 & 1.7 & 24.0 & 1.6 & -0.23 & $-1.09,0.64$ \\
\hline Intra-Abdominal AT & 10.6 & 1.2 & 11.1 & $1.4 * * *$ & 13.2 & 1.2 & 13.1 & 1.1 & -0.46 & $-0.87,-0.05$ \\
\hline Abdominal Subcutaneous AT & 23.1 & 0.9 & 22.7 & 0.8 & 21.9 & 0.7 & 21.6 & 0.7 & -0.23 & $-0.88,0.42$ \\
\hline
\end{tabular}

AT, adipose tissue. $* * P<0.01$ within-group, $* * * P<0.001$ within-group, $\dagger P<0.05$ between groups.

Significantly fewer subjects gained $\geqslant 3 \%(P=0.036)$ and $>5 \%(P=0.033)$ of their baseline body weight following propionate ester supplementation. Weight loss after 24 weeks was greater in the propionate ester group, but this effect was not significantly different between groups $(-1.02 \mathrm{~kg}[95 \% \mathrm{CI},-2.10$ to 0.04$]$ propionate ester vs. $0.38 \mathrm{~kg}$ [95\% CI, -0.95 to 1.72$]$ control; $P=0.099)$. The change in the distribution of intra-abdominal adipose tissue (AT) was significantly lower in the propionate ester group compared with control supplementation $(P=0.027)$. Furthermore, internal AT $(P=0.002)$ was significantly increased within the control group, but not in the propionate ester group.

In conclusion, this investigation provides the first direct evidence that colonic propionate can prevent body weight and intra-abdominal AT gain in humans. Dietary strategies that promote colonic propionate production may play a role in weight management over the life-course.

This work was supported by the Biotechnology \& Biological Sciences Research Council (BB/H004971/1). The clinical trial (NCT00750438) was conducted in accordance with the Declaration of Helsinki.

1. Tolhurst G, Heffron H, Lam YS, et al. (2011) Diabetes 61, 364-371. 